\title{
Loss of Caveolin 1 is Associated With the Expression of Aquaporin 1 and Bladder Dysfunction in Mice
}

\author{
Seheon Jung ${ }^{1}$, Sun-Ouck Kim ${ }^{1}$, Kyung-Aa Cho ${ }^{2}$, Seung Hee Song ${ }^{1}$, Teak Won Kang ${ }^{1}$, Kwangsung Park ${ }^{1}$, Dongdeuk Kwon ${ }^{1}$ \\ Departments of ${ }^{1}$ Urology and ${ }^{2}$ Biochemistry, Chonnam National University Hospital, Chonnam National University Medical School, Gwangju, Korea
}

CrossMark

Purpose: It is suggested that caveolin and aquaporin might be closely associated with bladder signal activity. We investigated the effect of the deletion of caveolin 1, using caveolin 1 knockout mice, on the expression of aquaporin 1 in order to identify their relation in the urothelium of the urinary bladder.

Methods: The cellular localization and expressions of aquaporin 1 and caveolin 1, in the wild type and caveolin 1 knockout mice urinary bladder, were examined by Western blot and immunofluorescence techniques.

Results: Aquaporin 1 and caveolin 1 were coexpressed in the arterioles, venules, and capillaries of the suburothelial layer in the wild type controls. Aquaporin 1 protein expression was significantly higher in the caveolin 1 knockout mice than in the wild type controls $(\mathrm{P}<0.05)$.

Conclusions: The results imply that aquaporin 1 and caveolin 1 may share a distinct relation with the bladder signal activity. This might play a specific role in bladder dysfunction.

Keywords: Aquaporin 1; Caveolin 1; Bladder

- Grant Support: This study was supported by Basic Science Research Program through the National Research Foundation of Korea (NRF) funded by the Ministry of Education, Science and Technology (2012-2139, 2013-2109), by a grant (CRI13005-1) from Chonnam National University Hospital Research Institute of Clinical Medicine and Research Institute of Medical Sciences, Chonnam National University (2013-CURIMS-DR002).

- Research Ethics: All mice procedures were performed according to the guidelines of the Animal Care and Use Committee of Chonnam National University. This study was permitted by the Ethics Committee of the Chonnam National University Medical School (CNU IACUCH-2009-80).

- Conflict of Interest: No potential conflict of interest relevant to this article was reported.

\section{INTRODUCTION}

The urothelium has conventionally been regarded as a boundary between urine and the urinary tract. However, a number of studies have shown that it also possesses properties that are characteristic of organs capable of perceiving diverse signals from the urinary bladder [1]. Thus, the urothelium can function as a target organ for neurotransmitters (from the urinary bladder), release chemical mediators, as well as respond to external stimuli by expressing sensory molecules [2].

Meanwhile, several studies on the role of aquaporins (AQPs) in the urothelium or smooth muscles of the urinary bladder have illustrated the possible relation between AQPs and bladder dysfunction [3-6]. AQPs are transmembrane proteins that are involved in ion or water transport across the cell membranes [7]. They are expressed in cells like those of the kidney, which have a functional role in high rates of water transport. Interestingly, though, they are also frequently found in cells not involved in
Corresponding author: Sun-Ouck Kim (iD http://orcid.org/0000-0002-5832-8303 Department of Urology, Chonnam National University Hospital, Chonnam National University Medical School, 42 Jebong-ro, Dong-gu, Gwangju 501-757, Korea E-mail: seinsena@hanmail.net / Tel: +82-62-220-6702 / Fax: +82-62-227-1643 Submitted: October 30, 2014 / Accepted after revision: March 7, 2015 (c) (7) This is an Open Access article distributed under the terms of the Creative Commons Attribution Non-Commercial License (http://creativecommons.org/licenses/by-nc/3.0/) which permits unrestricted non-commercial use, distribution, and reproduction in any medium, provided the original work is properly cited. 
active water transport, such as the vascular endothelium and smooth muscle cells $[7,8]$.

Flask-shaped caveolae are small invaginations of the cell membrane that are easily found in well-differentiated cells, such as those of the muscle. Caveolins (CAVs) are integral membrane proteins of the caveolae, and are essential for their formation. Moreover, these proteins have an important functional role in cell signaling, whereby they serve as scaffolds to foster the compartmentalization and integration of signals [9]. There are three isoforms of CAVs: CAV1, CAV2, and CAV3. Among them, CAV1 has been shown to play a role in the regulation of signal transduction pathways in a variety of cells [10]. Furthermore, Lai et al. [11] demonstrated that CAV1 knockout (KO) mice lack caveolae in the urinary bladder, and represent a number of urological disorders. In addition, CAV1 KO mice also exhibit decreased acetylcholine excretion and disrupted M3 muscarinic cholinergic activity of the bladder, leading to impaired smooth muscle contraction.

In our previous reports, we demonstrated altered expression of AQP1 in the urinary bladder of rats presenting detrusor overactivity [12-14]. Further, in a more recent study, we speculated about the relation between AQP1 and CAV1 in the urinary bladder, and found that they are significantly overexpressed upon detrusor overactivity (caused by bladder outlet obstruction $[\mathrm{BOO}])$. Moreover, AQP1 and CAV1 were found to colocalize in the suburothelial microvasculature [12-14]. Based on earlier observations, we suggested that AQP1 and CAV1 might be closely associated with bladder signal activity that may, in turn indicate their role in detrusor overactivity. To date, however, no study has assessed the interrelationship between AQPs and CAVs in the urinary bladder, using CAV KO mice models. Thus, the purpose of this study is to investigate the expression of AQP1 in wild type and CAV1 KO mice, in order to determine their potential roles in the urinary bladder. We have specifically focused on the suburothelium, since this is the current target for research on bladder dysfunction.

\section{MATERIALS AND METHODS}

\section{Animal Models}

Young female C57BL/6J mice (8-10 weeks old; $\mathrm{n}=10$ ), and CAV1 KO mice (backcrossed to C57BL/6J for 10 generations; $\mathrm{n}=10$ ) were obtained from the Jackson Laboratory (Bar Harbor, ME, USA). The mice were inbred by homozygous mating. All mice were maintained in a pathogen-free animal center at the
Clinical Vaccine Center of Chonnam National University (CNU IACUC-H-2009-80).

The mice were anesthetized with xylazine $(2.2 \mathrm{mg} / \mathrm{kg}$, intramuscularly) and zolazepam/tiletamine $(4.4 \mathrm{mg} / \mathrm{kg}$, intramuscularly). All mice procedures were performed according to the guidelines of the Animal Care and Use Committee of Chonnam National University. This study was permitted by the Ethics Committee of the Chonnam National University Medical School.

\section{Western Blot}

Tissues $(\mathrm{n}=10)$ were homogenized in ice-cold isolation solution with a Tissumizer homogenizer (Tekmar, Cincinnati, OH, USA) by a micro-saw tooth generator (five bursts, five strokes). Total protein was isolated from the homogenized tissues, and resolved by gel electrophoresis (12\% sodium dodecyl sulfatepolyacrylamide; $50 \mu \mathrm{g}$ of protein). They were then transferred to polyvinylidene difluoride membranes (Amersham Pharmacia Biotech Inc., Piscataway, NJ, USA). Subsequently, the membranes were washed with Tris-buffered saline with Tween 20 buffer (10-mM Tris-HCl, pH 7.6; 150-mM NaCl; 0.05\% Tween-20). They were then blocked with 5\% nonfat dry milk, and incubated with the respective primary antibodies: rabbit polyclonal anti-AQP1 antibody (1:500; Santa Cruz Biotechnology Inc., Dallas, TX, USA), mouse monoclonal anti-CAV1 (1:1,000; BD Biosciences, San Jose, CA, USA), and rabbit polyclonal antiglyceraldehyde 3-phosphate dehydrogenase (GAPDH) antibody (1:4,000; Cell Signaling Technology, Danvers, MA, USA). Horseradish peroxidase-conjugated antirabbit antibody for AQP1 (1:2,000; Santa Cruz Biotechnology Inc.) and GAPDH (1:4,000; Sigma, St. Louis, MO, USA) were the secondary antibodies used. Protein bands were detected by WESTZOL plus Western Blot Detection System (iNtRON Biotechnology Inc., Seongnam, Korea), and visualized with LAS-3000 (Fujifilm Life-science, Tokyo, Japan). Densitometric scanning was conducted by the Multi gauge V3.0 (Fujifilm Life-science) analysis software and chemiluminescence system.

\section{Immunofluorescence Staining}

The tissue sections (10 for each tissue) were washed in phosphate buffered saline, and incubated with normal chicken serum (3\%, 30 minutes, room temperature) to prevent nonspecific binding. They were then incubated with antibodies against AQP1 (1:100; Santa Cruz Biotechnology Inc.) and CAV1 (1:100; BD Biosciences) for $12-14$ hours at $4^{\circ} \mathrm{C}$. Immunoreactivity for 
CAV1 was detected using Alexa Fluor 594 chicken antimouse $\operatorname{IgG}(\mathrm{H}+\mathrm{L})$, and for AQP1 by Alexa Fluor 594 chicken antirabbit $\operatorname{IgG}(\mathrm{H}+\mathrm{L})$ (1:100; Molecular Probes Inc., Eugene, OR, USA). DAPI (4'-6-diamidino-2-phenylindole) (Vector Laboratories, Burlingame, CA, USA) was used for tissue mounting, to stain the nucleus. For negative controls, tissues were similarly processed, but without AQP1 and CAV1 antibodies. Tissues were visualized with a confocal microscope (LSM 510, Carl Zeiss Korea, Seoul, Korea) with wavelengths appropriate for Alexa Fluor $(594 \mathrm{~nm})$ and Alexa Fluor (405 nm). Densitometric analysis was performed with Studio Star Scanner using the NIH Image V157 software.

\section{Statistical Analysis}

The results were expressed as the mean \pm standard deviation. Mann-Whitney test was used for statistical analysis. A statistical significance was considered at $\mathrm{P}<0.05$.

\section{RESULTS}

There was no difference in body weights $(24.2 \pm 0.4 \mathrm{~g}$ vs. $23.9 \pm$ $0.5 \mathrm{~g})$ and bladder weights $(44.8 \pm 1.2 \mathrm{mg}$ vs. $45.1 \pm 0.8 \mathrm{mg})$ between the two groups (control vs. CAV1 KO).

\section{Effect of CAV1 KO on AQP1 Expression, as Revealed by Immunofluorescence}

AQP1 and CAV1 were observed to be coexpressed in the capillaries, venules, and arterioles of the suburothelial layer of the wild type tissues (Fig. 1). The immunofluorescence study further revealed that the expression of AQP1 was higher in the CAV1 KO mice than in the wild type controls (Fig. 1).

\section{Expression of AQP1 in CAV1 KO Mice, as Detected by Western Blotting}

Western blot analysis revealed bands for AQP1 and CAV1 at 28 $\mathrm{kDa}$ and $22 \mathrm{kDa}$, respectively (Fig. 2). Both the proteins were detected in the wild type group, whereas CAV1 was not detected in the CAV1 KO mice. Moreover, the expression of AQP1 was significantly increased in the CAV1 KO mice $(\mathrm{P}<0.05)$ (Fig. 2), in corroboration with the immunofluorescence data.

\section{DISCUSSION}

The present study demonstrated increased expression of AQP1 in the suburothelial microvasculature, in CAV1 KO mice uri-

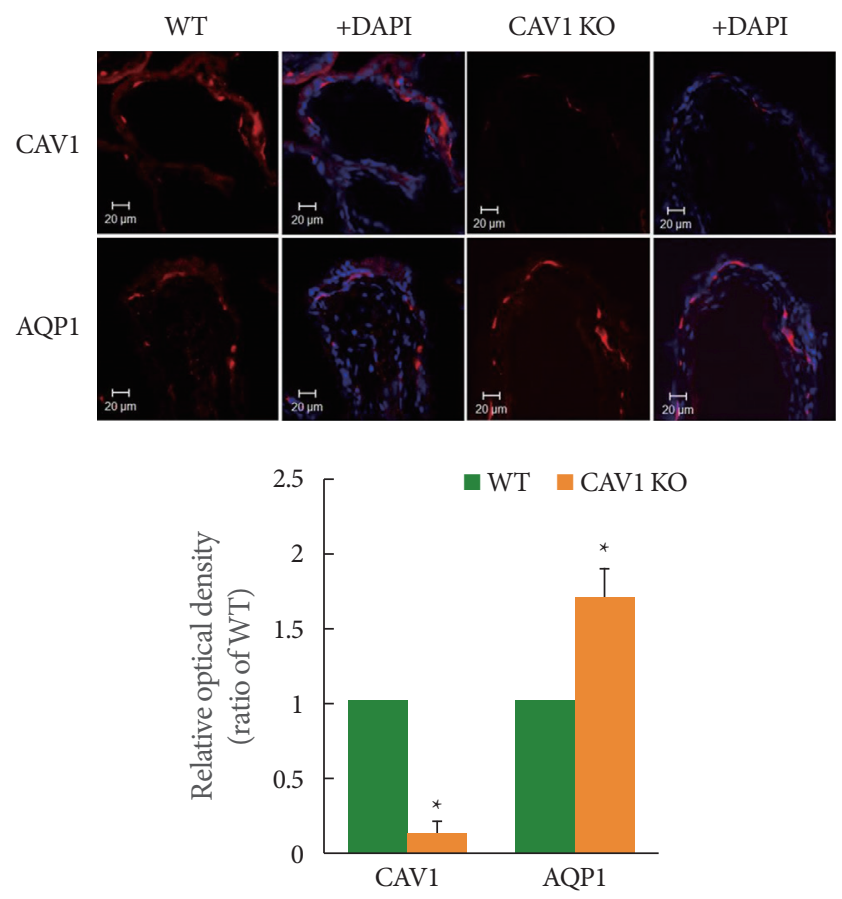

Fig. 1. Immunofluorescence labeling for aquaporin 1 (AQP1) and caveolin 1 (CAV1) in the wild type and CAV1 knockout (KO) mice urinary bladder. The expression of AQP1 and CAV1 was noted throughout the capillaries, venules, and vascular smooth muscle. The expression of AQP1 was increased in the CAV1 KO mice. The horizontal scale bar at the bottom left of each figure indicates the magnification power. The each lower panel denotes the means \pm standard deviation of experiments for each condition determined by relative densitometry. WT, wild type; DAPI, 4',6-diamidino-2-phenylindole. ${ }^{\star} \mathrm{P}<0.05$.

nary bladder. The immunohistochemical study showed that AQP1 and CAV1 in the urinary bladder colocalized in the arterioles, venules and capillaries of the suburothelial layer. Collectively, these results provide evidence that mice lacking CAV1 significantly overexpress AQP1 at the urothelium, which is actively involved in afferent signal transduction. Consequently, AQP1 and CAV1 might be closely associated, and may play a role in bladder dysfunction. To the best of our knowledge, this is the first in vivo study that demonstrates the possible manifestation of AQP1/CAV1-mediated suburothelial cell signaling in bladder dysfunction.

Various studies have implicated that the urothelium can mediate solute and water transport under specific circumstances $[15,16]$. The epithelial sodium channel (ENaC) is well known to be responsible for fluid and salt transport across epithelial cell membranes. Investigations have revealed that $\mathrm{ENaC}$ shows sig- 

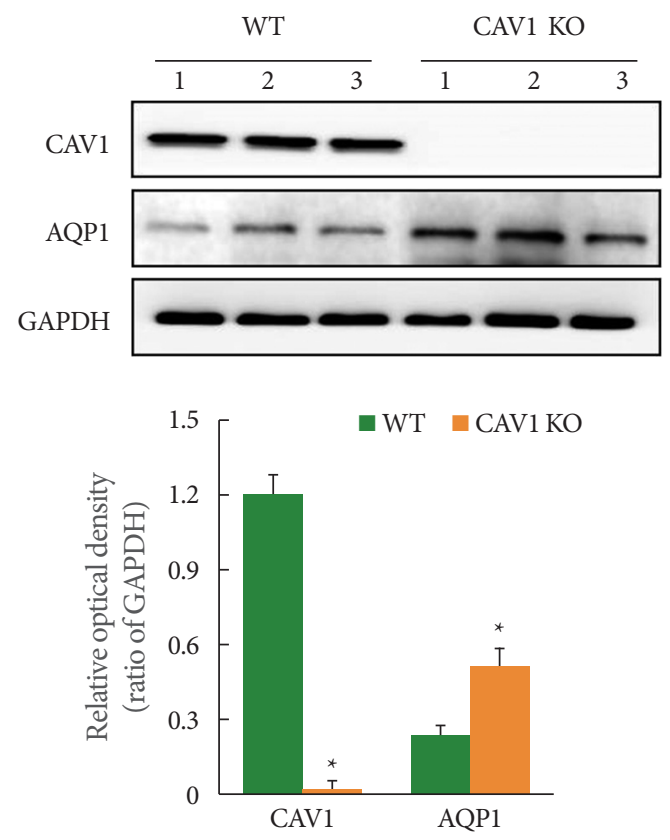

Fig. 2. Immunoblotting of aquaporin 1 (AQP1) and caveolin 1 (CAV1) in the wild type and CAV1 knockout (KO) mice urinary bladder. The anti-AQP antibodies recognize the $27-$ to $29-\mathrm{kDa}$ bands that correspond to glycosylated AQPs. The anti-CAV1 antibodies recognize the $22-\mathrm{kDa}$ band. Anti-GAPDH antibody recognizes the $42-\mathrm{kDa}$ band. The expression of AQP1 was significantly increased in the CAV1 KO mice. The lower panel denotes the means \pm standard deviation of the 10 experiments for each condition, as determined by the densitometry relative to GAPDH. WT, wild type; GAPDH, glyceraldehyde 3-phosphate dehydrogenase. ${ }^{\star} \mathrm{P}<0.05$.

nificantly greater expression in BOO patients with clinical symptoms of bladder dysfunction than in the controls. Clinically, this correlated with the storage symptom score of the patients [17]. However, studies on the expression of AQPs in the urinary bladder are limited. In our previous report, detrusor overactivity caused by BOO led to a significant increase in the expression of AQP2-3, and nitric oxide synthase (endothelial nitric oxide synthase, neuronal nitric oxide synthase) in rat urinary bladder. In the study, we suggested that the AQPs and nitric oxide synthase isoforms might have a specific role in bladder dysfunction that is related with bladder change following $\mathrm{BOO}$ [12].

The CAV1 KO mouse has been proposed as a novel animal model to study bladder impairment associated with primary hypocontraction of urinary bladder and detrusor overactivity $[11,18]$. Studies on CAV1 KO mice have established that loss of CAV1 is associated with disrupted muscarinic cholinergic activity. This induces impaired smooth muscle contraction in the urinary bladder upon stimulation with carbachol $[19,20]$. Further, in the organ bath study using bladder strips of CAV1 KO mice (produced by genetic ablation of caveolae), a 70\% decrease in acetylcholine release from bladder nerve terminals was observed [11]. However, the functional importance of these organelles in relation to the micturition response in the urinary bladder has not been entirely delineated.

Intriguingly, until date, there are no studies either on the relation between AQPs and CAVs in the suburothelium (with regard to bladder dysfunction), or the alteration of their expressions in CAV1 KO mice. Gao et al. [21], however, had revealed a functional interaction between AQP1 and CAV1 in the change of microvasculature and regulation of alveolar epithelial permeability in rat lung. They reported that CAV1 knock down by small interfering RNA causes increased microvascular and epithelial permeability as well as pulmonary edema. They also proposed that CAV1 plays an important role in the regulation of pulmonary permeability by modifying AQP1 [21].

It is important, however, to note the limitations of this study: Our hypothesis warrants in-depth studies showing (1) the physiological alteration of the urinary bladder. to confirm the CAV1/ AQP1 association at the suburothelium; (2) the functional signal change(s), or change in solute or water movement; and (3) specific functional changes in bladder activities. Deeper understanding of the AQP1/CAV1 relation is likely to explain the cellular mechanisms of bladder dysfunction, and may suggest potential molecular targets for its pharmacological treatment.

In conclusion, our results imply that lack of CAV1 may result in significant upregulation of AQP1, providing possible evidence that these proteins are closely associated in the urothelium. This is probably via modification of cellular transmission and signaling pathway(s) specific to the suburothelial tissue. The possible interrelation between AQP1 and CAV1 also stems from the fact that they colocalized at the endothelium of the microvasculature, which is important in terms of blood supply to the urothelial tissues.

\section{REFERENCES}

1. de Groat WC. The urothelium in overactive bladder: passive bystander or active participant? Urology 2004;64(6 Suppl 1):7-11.

2. Birder LA, de Groat WC. Mechanisms of disease: involvement of the urothelium in bladder dysfunction. Nat Clin Pract Urol 2007;4: 46-54.

3. Kim SO, Song SH, Hwang EC, Oh KJ, Ahn K, Jung SI, et al. Chang- 
es in aquaporin $(\mathrm{AQP}) 2$ and $\mathrm{AQP} 3$ expression in ovariectomized rat urinary bladder: potential implication of water permeability in urinary bladder. World J Urol 2012;30:207-12.

4. Kim SO, Song SH, Hwang EC, Park KS, Kwon DD, Ahn KY, et al. The expression of AQP1 and eNOS in menopausal rat urinary bladder. Int Neurourol J 2010;14:78-85.

5. Kim SO, Song SH, Ahn K, Kwon D, Park K, Ryu SB. Changes in aquaporin 1 expression in rat urinary bladder after partial bladder outlet obstruction: preliminary report. Korean J Urol 2010;51:281-6.

6. Verkman AS. More than just water channels: unexpected cellular roles of aquaporins. J Cell Sci 2005;118(Pt 15):3225-32.

7. Gannon BJ, Warnes GM, Carati CJ, Verco CJ. Aquaporin-1 expression in visceral smooth muscle cells of female rat reproductive tract. J Smooth Muscle Res 2000;36:155-67.

8. Matsuzaki T, Tajika Y, Ablimit A, Aoki T, Hagiwara H, Takata K. Aquaporins in the digestive system. Med Electron Microsc 2004;37: 71-80.

9. Smart EJ, Ying YS, Donzell WC, Anderson RG. A role for caveolin in transport of cholesterol from endoplasmic reticulum to plasma membrane. J Biol Chem 1996;271:29427-35.

10. Razani B, Lisanti MP. Caveolin-deficient mice: insights into caveolar function human disease. J Clin Invest 2001;108:1553-61.

11. Lai HH, Boone TB, Yang G, Smith CP, Kiss S, Thompson TC, et al. Loss of caveolin-1 expression is associated with disruption of muscarinic cholinergic activities in the urinary bladder. Neurochem Int 2004;45:1185-93.

12. Kim SO, Choi D, Song SH, Ahn KY, Kwon D, Park K, et al. Effect of detrusor overactivity on the expression of aquaporins and nitric oxide synthase in rat urinary bladder following bladder outlet obstruction. Can Urol Assoc J 2013;7:E268-74.
13. Kim SO, Song SH, Lee SC, Cho KA, Kim HS, Hwang IS, et al. Effects of estrogens on the expression of caveolin-1 in the urinary bladders of female rats. Int Neurourol J 2011;15:13-8.

14. Kim SO, Song SH, Lee SC, Cho KA, Yu HS, Hwang IS, et al. Expression of caveolin-1 in rat urinary bladder with cyclophosphamide-induced cystitis. Int Neurourol J 2012;16:169-74.

15. Negrete HO, Lavelle JP, Berg J, Lewis SA, Zeidel ML. Permeability properties of the intact mammalian bladder epithelium. Am J Physiol 1996;271(4 Pt 2):F886-94.

16. Cross WR, Eardley I, Leese HJ, Southgate J. A biomimetic tissue from cultured normal human urothelial cells: analysis of physiological function. Am J Physiol Renal Physiol 2005;289:F459-68.

17. Araki I, Du S, Kamiyama M, Mikami Y, Matsushita K, Komuro M, et al. Overexpression of epithelial sodium channels in epithelium of human urinary bladder with outlet obstruction. Urology 2004; 64:1255-60.

18. Woodman SE, Cheung MW, Tarr M, North AC, Schubert W, Lagaud G, et al. Urogenital alterations in aged male caveolin-1 knockout mice. J Urol 2004;171(2 Pt 1):950-7.

19. Cao G, Yang G, Timme TL, Saika T, Truong LD, Satoh T, et al. Disruption of the caveolin-1 gene impairs renal calcium reabsorption and leads to hypercalciuria and urolithiasis. Am J Pathol 2003;162: 1241-8.

20. Lai HH, Boone TB, Thompson TC, Smith CP, Somogyi GT. Using caveolin-1 knockout mouse to study impaired detrusor contractility and disrupted muscarinic activity in the aging bladder. Urology 2007;69:407-11.

21. Gao C, Li R, Huan J, Li W. Caveolin-1 siRNA increases the pulmonary microvascular and alveolar epithelial permeability in rats. J Trauma 2011;70:210-9. 